\title{
Progressive systemic sclerosis complicated by diffuse pulmonary haemorrhage
}

\author{
J. KALLENBACH, I. PRINSLOO, AND S. ZWI
}

From the Respiratory Unit, Department of Medicine, University of the Witwatersrand and Johannesburg Hospital, and National Research Institute for Occupational Diseases of the South African Medical Research Council, Johannesburg, South Africa

Kallenbach, J., Prinsloo, I., and Zwi, S. (1977). Thorax, 32, 767-770. Progressive systemic sclerosis complicated by diffuse pulmonary haemorrhage. A case is reported of progressive systemic sclerosis with pulmonary fibrosis which was complicated by recurrent haemoptyses due to diffuse pulmonary haemorrhage. We have found no other report of this association. The haemorrhage finally remitted after treatment was started with $40 \mathrm{mg}$ prednisone daily, though previously spontaneous remissions had occurred.

We report a known case of progressive systemic sclerosis with pulmonary fibrosis who presented with recurrent haemoptyses. Haemoptysis in this situation may indicate the presence of a malignant lung tumour and should, therefore, be thoroughly investigated (Tomkin, 1969). The clinical course and the results of our investigations in this patient suggest that the haemoptysis was due to diffuse pulmonary haemorrhage (Thomas and Irwin, 1975).

\section{Case report}

A 48-year-old white man was diagnosed as having scleroderma in 1951. Skin biopsy was compatible with the clinical diagnosis. He was well until 1970 , when he began to have symptoms of Raynaud's phenomenon and mild dyspnoea on exertion. A chest radiograph showed a basal interstitial infiltrate, and pulmonary function tests revealed evidence of a mild restrictive defect. The pulmonary changes were attributed to systemic sclerosis. He was treated with small oral doses of a corticosteroid preparation, but mild dyspnoea persisted, causing slight limitation of activity.

In December 1975 the patient began to have episodes of haemoptysis, productive of about $10 \mathrm{ml}$ blood daily, associated with a significant increase in dyspnoea. He was admitted to the Johannesburg Hospital in January 1976. He was apyrexial but pale with a respiratory rate of $36 / \mathrm{min}$ and a regular heart rate of $120 / \mathrm{min}$. The blood pressure was $110 / 70 \mathrm{mmHg}$. The hands showed evidence of scleroderma with sclerodactyly and telangiectases, and the mouth was contracted. Extensive bilateral basal crepitations were audible in the lungs. There was no evidence of left-sided cardiac failure or any other cardiac abnormality.

The ECG was normal. A chest radiograph showed extensive bilateral alveolar consolidation (Fig. 1). A barium swallow showed no evidence of oesophageal abnormality. Laboratory investigations revealed: $\mathrm{Hb} 9 \mathrm{~g} / \mathrm{dl}$ with hypochromia and microcytosis of the red cells; white cell count $8.9 \times 10^{9} / 1$ with a normal differential count; blood urea $8 \mathrm{mmol} / 1$ (48 $\mathrm{mg} / 100 \mathrm{ml})$ with normal electrolytes; serum creatinine $110 \mu \mathrm{mol} / 1$ (1.2 mg/ $100 \mathrm{ml})$; serum iron $9 \mu \mathrm{mol} / 1(47 \mu \mathrm{g} / 100 \mathrm{ml})$ with a transferrin level of $48 \mu \mathrm{mol} / 1(265 \mu \mathrm{g} / 100 \mathrm{ml})$; antinuclear factor positive with a titre of $1: 640$; serum complement normal. Antiglomerular basement membrane antibodies (AGBMA) were not detected in the serum. Urine examination was normal.

Fibreoptic bronchoscopy was performed and revealed no abnormality. Transbronchial lung biopsy yielded fragments of tissue which were too small for accurate histological assessment. Pulmonary function tests again showed the features of a mild restrictive defect with a $\mathrm{Po}_{2}$ of $8 \mathrm{kPa}(60 \mathrm{mmHg})$ [normal value for Johannesburg, altitude $1760 \mathrm{~m}$ : $10 \mathrm{kPa}(75 \mathrm{mmHg})]$.

The patient's haemoptysis subsided gradually, and his dyspnoea improved. The radiological picture improved simultaneously until only a basal interstitial infiltrate remained. A tentative diag- 


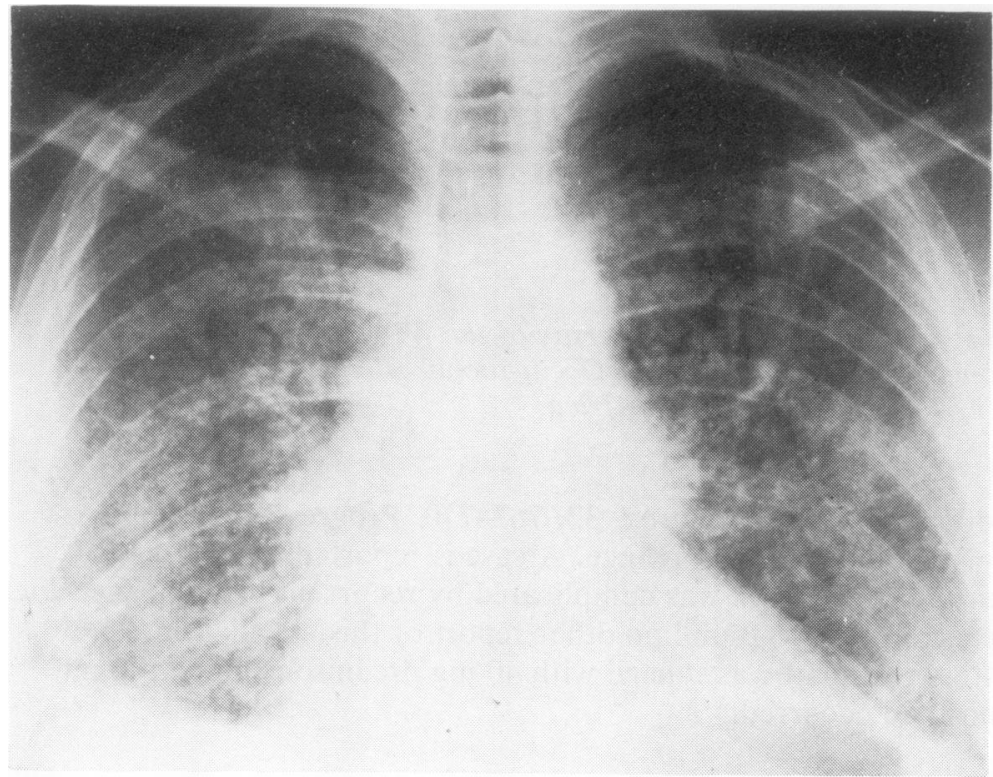

Fig. 1 Chest radiograph January 1976, showing bilateral alveolar consolidation. nosis of diffuse pulmonary haemorrhage was made (Thomas and Irwin, 1975). Therapy with corticosteroids was changed to prednisone, $10 \mathrm{mg} / \mathrm{day}$. In February and August 1976 the patient experienced further episodes of haemoptysis, each with increasing dyspnoea, a fall in the haemoglobin level, and the appearance of diffuse alveolar consolidation on the chest radiograph. The clinical findings were the same as on the previous occasion. These episodes resolved in hospital without any change of therapy.

In October 1976 the patient had a severe exacerbation with a fall in the haemoglobin to $6 \mathrm{~g} / \mathrm{dl}$, necessitating blood transfusion. Repeat bronchoscopy showed no abnormality. Open lung biopsy was therefore performed. Histology showed honeycomb formation with replacement of the normal lung parenchyma with fibrous tissue. There was an increase of connective tissue in the alveolar walls. The alveoli contained desquamated cells and, in addition, intact red blood cells and haemosiderin-laden macrophages. The pulmonary arteries showed intimal thickening but there was no evidence of acute vasculitis. The findings were compatible with pulmonary fibrosis associated with progressive systemic sclerosis complicated by diffuse haemorrhage (Fig. 2).

Treatment was begun with prednisone, $40 \mathrm{mg}$ daily. The haemoptysis again subsided with improvement in the patient's dyspnoea and clearing of the chest radiograph. Therapy has been continued with prednisone in a dosage of $80 \mathrm{mg}$ on alternate days, and he has remained asymptomatic except for mild dyspnoea. The current chest radiograph again shows only a basal interstitial infiltrate (Fig. 3). Repeated tests for AGBMA in the serum have been negative, the urea and creatinine levels have remained unchanged, and examination of the urine has been normal at all times up to the present (April 1977).

\section{Discussion}

Diffuse pulmonary haemorrhage in the absence of renal disease, AGBMA, vasculitis, or left-sided cardiac failure has been designated idiopathic pulmonary haemosiderosis (IPH) (Thomas and Irwin, 1975). Despite the absence of all these factors in our patient we prefer to avoid the use of this term because of its association with a defined disease entity, namely, progressive systemic sclerosis. The pathogenic mechanism in most cases of diffuse pulmonary haemorrhage is uncertain. A number of different abnormalities have been implicated. Soergel and Sommers (1962) suggested that IPH was a distinct clinical and pathological entity due to an abnormality of pulmonary alveolar epithelial growth and function resulting directly in alveolar capillary haemorrhages. On the other hand, the association of diffuse pulmonary haemorrhage with glomerulonephritis mediated by AGBMA (Goodpasture's syndrome) suggests that there may be a disease continuum involving pulmonary capillaries and the glomerular basement 


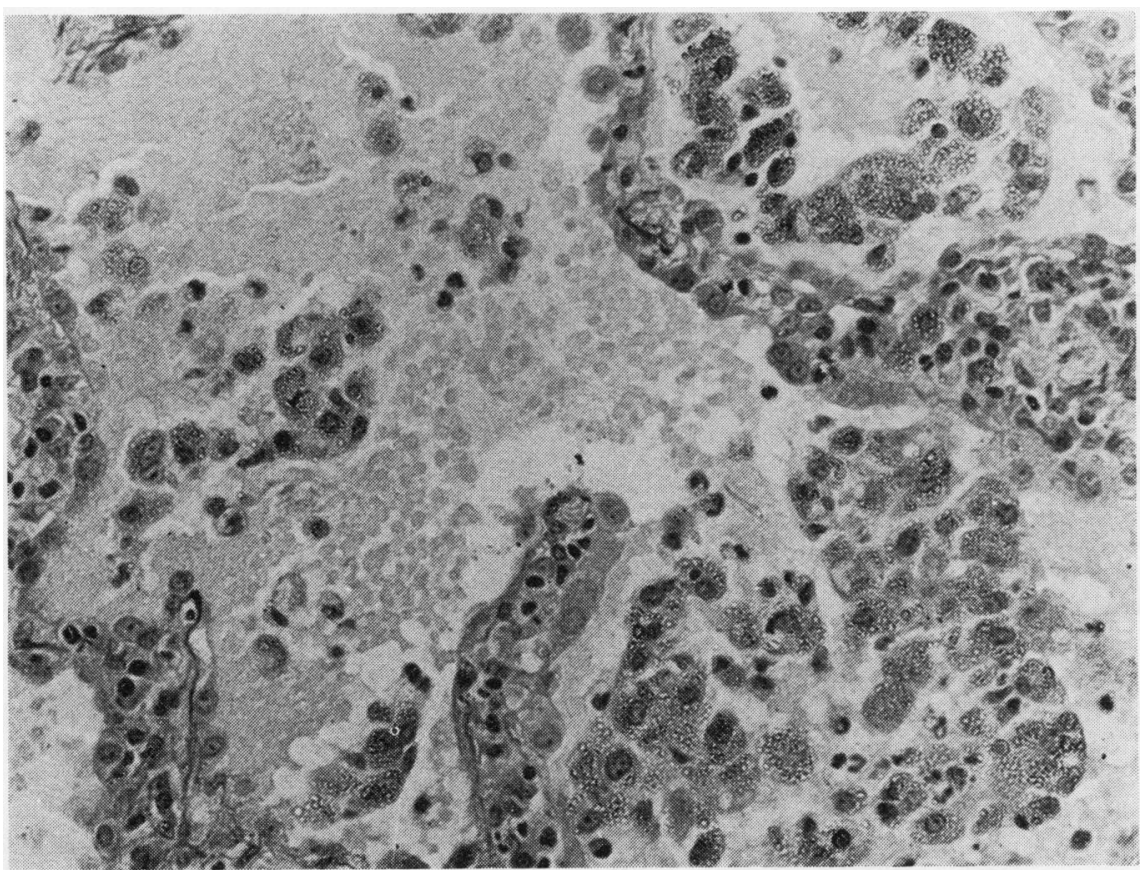

Fig. 2 Section of lung showing alveoli containing numerous red blood cells and haemosiderin-laden macrophages (Haematoxylin, eosin, and aldehyde fuchsin $\times 210$ ).

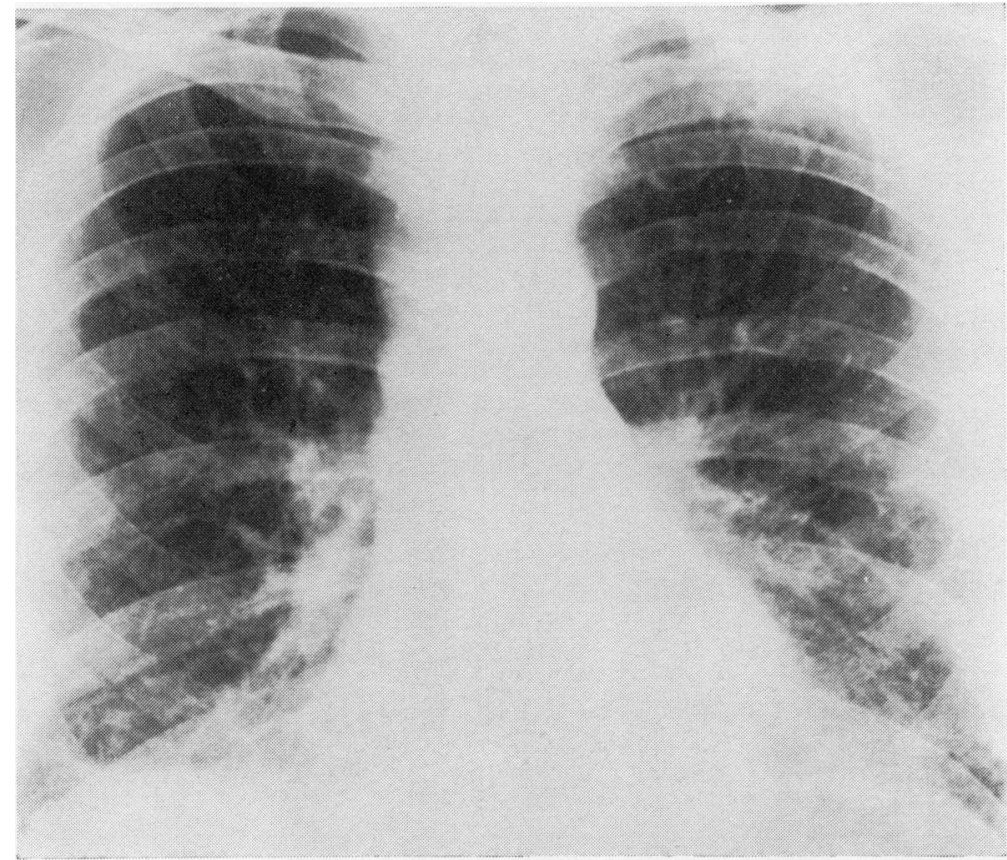

Fig. 3 April 1977, showing

basal interstitial infiltrate only. 
membrane and having an immunological basis (Elder et al., 1965). Moreover, pulmonary haemorrhage may be the presenting feature in Goodpasture's syndrome, and only the advent of manifestations of renal disease, or the detection of AGBMA in the serum up to one year later (Wilson and Dixon, 1973; 1974), will make the correct diagnosis possible.

The literature contains references to a number of cases described as IPH occurring in association with connective tissue diseases and immunological disorders. An association with rheumatoid arthritis has been found in a number of cases (Karlish, 1962; Ognibene and Dito, 1965; Smith, 1966). Two cases have been reported in which features of lupus erythematosus occurred five years and 18 months after a diagnosis of IPH had been made (Byrd and Trunk, 1973; Kuhn, 1972). O'Donohue (1974) described a case in which features suggestive of IPH were followed by arthritis, immune complex nephritis, and, finally, features compatible with the diagnosis of Wegener's granulomatosis. There are also reports (Beirne et al., 1973; Lewis et al., 1973; Clinicopathologic conference, 1973) of pulmonary haemorrhage occurring in the presence of immune complex nephritis as opposed to the linear deposits of antibodies characteristic of Goodpasture's syndrome. We have found no other report of diffuse pulmonary haemorrhage in a case of progressive systemic sclerosis, and this case therefore should be added to the list of connective tissue disorders reported to have been complicated by this occurrence. These associations suggest that the pulmonary haemorrhage may, occasionally, be a manifestation of a systemic connective tissue or immunological disorder.

Treatment with corticosteroids has been reported to cause remission in cases of IPH (Soergel and Sommers, 1962). Although the haemorrhage has been controlled in our patient since the introduction of a relatively large dosage of prednisone, it is possible that this is due merely to a fluctuation in the natural course of the disease.

\section{References}

Beirne, G. J., Kopp, W. L., and Zimmerman, S. W. (1973). Goodpasture syndrome: dissociation from antibodies to glomerular basement membrane. Archives of Internal Medicine, 132, 261-263.
Byrd, R. B., and Trunk, G. (1973). Systemic lupus erythematosus presenting as pulmonary hemosiderosis. Chest, 64, 128-129.

Clinicopathologic conference (1973). Proliferative glo- $\overparen{\mathbb{D}}$ merulonephritis and pulmonary hemorrhage. American Journal of Medicine, 55, 199-210.

Elder, J. L., Kirke, G. M., and Smith, W. G. (1965). $\overrightarrow{0}$ Idiopathic pulmonary haemosiderosis and the Good- pasture syndrome. British Medical Journal, 2, 1152- $\vec{\omega}$ 1155.

Karlish, A. J. (1962). Idiopathic pulmonary haemo- $\vec{x}$ siderosis with unusual features. Proceedings of the Royal Society of Medicine, 55, 223-225.

Kuhn, C. (1972). Systemic lupus erythematosus in o patient with ultrastructural lesions of the pulmon- of ary capillaries previously reported in the Review as $O$ due to idiopathic pulmonary hemosiderosis (letter). American Review of Respiratory Disease, 106, 931932.

Lewis, E. J., Schur, P. H., Busch, G. J., Galvanek, E., and Merrill, J. P. (1973). Immunopathologic features 3 of a patient with glomerulonephritis and pulmonary $\mathbb{\Phi}$ hemorrhage. American Journal of Medicine, 54, $507-513$.

O'Donohue, W. J., Jr. (1974). Idiopathic pulmonary. hemosiderosis with manifestations of multiple connective tissue and immune disorders. Treatment with cyclophosphamide. American Review of Respiratory Disease, 109, 473-479.

Ognibene, A. J., and Dito, W. R. (1965). Rheumatoid $\frac{\varnothing}{\varnothing}$ disease with unusual pulmonary manifestations. @ Archives of Internal Medicine, 116, 567-572.

Smith. B. S. (1966). Idiopathic pulmonary haemosiderosis and rheumatoid arthritis. British Medical Journal, 1, 1403-1404.

Soergel, K. H., and Sommers, S. C. (1962). Idiopathic pulmonary hemosiderosis and related syndromes. American Journal of Medicine, 32, 499-511.

Thomas. H. M., and Irwin, R. S. (1975). Classification of diffuse intrapulmonary hemorrhage. Chest, 68, 483-484.

Tomkin, G. H. (1969). Systemic sclerosis associated with carcinoma of the lung. British Journal of Dermatology, 81, 213-216.

Wilson, C. B., and Dixon, F. J. (1973). Antiglomerular basement membrane antibody-incluced glomerulonephritis. Kidney International, 3, 74-89.

Wilson, C. B., and Dixon, F. J. (1974). Diagnosis of $\widehat{N}$ immunopathologic renal disease. Kidney Inter- N national. 5, 389-401.

Requests for reprints to: Professor S. Zwi, Depart-c ment of Medicine, Medical School, Hospital Street, $\underset{\mathbb{C}}{\stackrel{C}{ }}$ Johannesburg 2001, South Africa. 